\title{
Multifractal Burst in the Spatiotemporal Dynamics of Jerky Flow
}

\author{
M. S. Bharathi, ${ }^{1}$ M. Lebyodkin, ${ }^{2}$ G. Ananthakrishna, ${ }^{1, *}$ C. Fressengeas,${ }^{3}$ and L. P. Kubin ${ }^{4}$ \\ ${ }^{1}$ Materials Research Centre, Indian Institute of Science, Bangalore 560 012, India \\ ${ }^{2}$ Institute of Solid State Physics, Russian Academy of Science, 142432 Chernogolovka, Moscow district, Russia \\ ${ }^{3}$ Laboratoire de Physique et Mécanique des Matériaux, Université de Metz, Ile du Saulcy, 57045 Metz Cedex 01, France \\ ${ }^{4}$ Laboratoire d'Etude des Microstructures, CNRS-ONERA, 29 Avenue de la Division Leclerc, 92322 Chatillon Cedex, France
}

\begin{abstract}
The collective behavior of dislocations in jerky flow is studied in Al-Mg polycrystalline samples subjected to constant strain rate tests. Complementary dynamical, statistical, and multifractal analyses are carried out on the stress-time series recorded during jerky flow to characterize the distinct spatiotemporal dynamical regimes. It is shown that the hopping type $B$ and the propagating type $A$ bands correspond to chaotic and self-organized critical states, respectively. The crossover between these types of bands is identified by a large spread in the multifractal spectrum. These results are interpreted on the basis of competing scales and mechanisms.
\end{abstract}

Jerky flow or the repeated yielding of alloys during plastic deformation has been studied since the turn of the last century [1], and still continues to engage the attention of scientists. The Portevin-Le Chatelier effect (PLC), as it is referred to, has been observed in many dilute alloys [2]. It is one of the few striking examples of the complexity of the spatiotemporal dynamics arising from the collective behavior of defect populations. In a tension test with a constant imposed strain rate $\dot{\epsilon}_{a}$, in practice a constant pulling velocity $V$, the effect manifests itself as a series of serrations in the stress-time or strain curve. Each stress drop is associated with the nucleation of a band of localized plastic deformation which under certain conditions propagates along the sample. The continued interest in the PLC effect arises not only from its intriguing spatiotemporal behavior but also from its detrimental influence on the mechanical properties of materials. Our purpose is to quantify the connection between the spatial (types of deformation bands) and temporal (nature of serrations) manifestations of the phenomenon.

It is now accepted [2,3] that the microscopic origin of the PLC effect is the dynamic aging of the material due to the interaction between mobile dislocations and diffusing solute atoms. In a certain range of strain rates and temperatures, the diffusion time of the solute atoms is of the order of the waiting time of the dislocations temporarily arrested at obstacles. Then, solute atoms can diffuse to and age the dislocations. Thus, the stress needed to unpin the dislocations increases with increasing waiting time and decreasing strain rate. At the macroscopic scale, this inverse force versus flux relation translates into a negative sensitivity of the flow stress to the strain rate. When the imposed strain rate falls into this range, the plastic deformation becomes nonuniform.

In polycrystals, the metallurgical taxonomy distinguishes three generic types of serrations, namely types $A, B$, and $C$ [4]. On increasing strain rates or decreasing the temperature, one first finds the type $C$, identified with randomly nucleated static bands and large characteristic stress drops. Then the type $B$ with smaller serration amplitudes is found. The bands formed are still localized and static in nature, but forming ahead of the previous band in a spatially correlated way giving the visual impression of a hopping propagation. Finally, one observes continuously propagating type $A$ bands associated with small stress drops. The decrease in the amplitude of the stress drop with increasing $\dot{\epsilon}_{a}$ reflects the decrease in the solute concentration on the arrested dislocations.

This wealth of spatiotemporal features has long defied a proper understanding. Renewed interest has come from the connections made to nonlinear dynamics. Dynamical [5-7] and statistical methods [4,7-9] have recently been applied to characterize the complexity of the stress-time series. Studies in Refs. [4-8] suggest that two distinct dynamical regimes encountered in single crystals might also be found in polycrystals. One is the chaotic dynamics [7] characterized by only a few degrees of freedom, identified as stress and dislocation densities [10]. The other is self-organized critical (SOC) dynamics [7], involving infinite degrees of freedom [11]. In polycrystals, only chaotic dynamics has been identified so far [6].

This paper is focused on type $B$ and $A$ bands in polycrystalline samples as they are expected to show interesting dynamical behavior in contrast to the uncorrelated type $C$ bands not considered here. We propose to identify the different dynamical regimes and their correlations with type $B$ and $A$ bands in polycrystalline samples. To unravel this missing connection, dynamical, statistical, and multifractal analyses of the stress-time series are combined with the identification of the spatial band patterns. The focus is on the multifractal analysis. The motivation for using this method stems from a conceptual similarity between the present transition from hopping to propagating bands and the Anderson transition in disordered systems [12]. In the Anderson model [13], wave functions are localized when the energy, $E$, is below the mobility edge $E_{c}$ and extended 
for $E>E_{c}$. At $E=E_{c}$, the states are shown to exhibit a multifractal character [13]. In the PLC effect, the hopping type $B$ bands are essentially localized, whereas type $A$ bands are delocalized in the sense that they are propagating. It is this crossover from localized to delocalized nature of the bands that we wish to capture by multifractal analysis. We identify the dynamical regimes associated with types $B$ and $A$ bands as chaotic and SOC type, respectively, and show that the range of multifractality exhibits a sharp peak in the transition region. This is shown to be a consequence of a few relevant competing scales and mechanisms.

The tensile samples of an $\mathrm{Al}-2.5 \% \mathrm{Mg}$ alloy, of length $L=70 \mathrm{~mm}$, were cut out of a polycrystalline cold-rolled sheet, parallel to the rolling direction. The grains were anisotropic in shape, with an aspect ratio of 5. Tests were carried out at room temperature for eight values of $\dot{\epsilon}_{a}=$ $V / L$ in the range of type $B$ and $A$ bands, from $5.56 \times$ $10^{-6}$ to $1.4 \times 10^{-2} \mathrm{~s}^{-1}$. The stress was recorded at a sampling rate of $20-200 \mathrm{~Hz}$. Three typical stress-time, $\sigma(t)$, curves (corrected for the drift due to strain hardening) are shown in Fig. 1 together with $|d \sigma / d t|$.

The dynamical analysis starts by embedding the time series in a higher dimensional space using the time delay technique. Consider the stress signal $\{\sigma(k), k=1$, $2, \ldots, K\}$, where $K$ is the number of data points and $k$ is in units of the sampling time. Then, a $d$-dimensional vector is defined by $\vec{\xi}_{k}=\{\sigma(k), \sigma(k+\tau), \ldots, \sigma[k+$ $(d-1) \tau]\}, k=1, \ldots,[K-(d-1) \tau]$, where $\tau$ is a delay time (in units of the sampling time). The set $\left\{\vec{\xi}_{k}, k=1, \ldots,[K-(d-1) \tau]\right\}$ constitutes the reconstructed attractor. The correlation dimension, $\nu$, is obtained by using the Grassberger-Procaccia algorithm [14]. In this method one calculates the correlation integral $C(r)$,

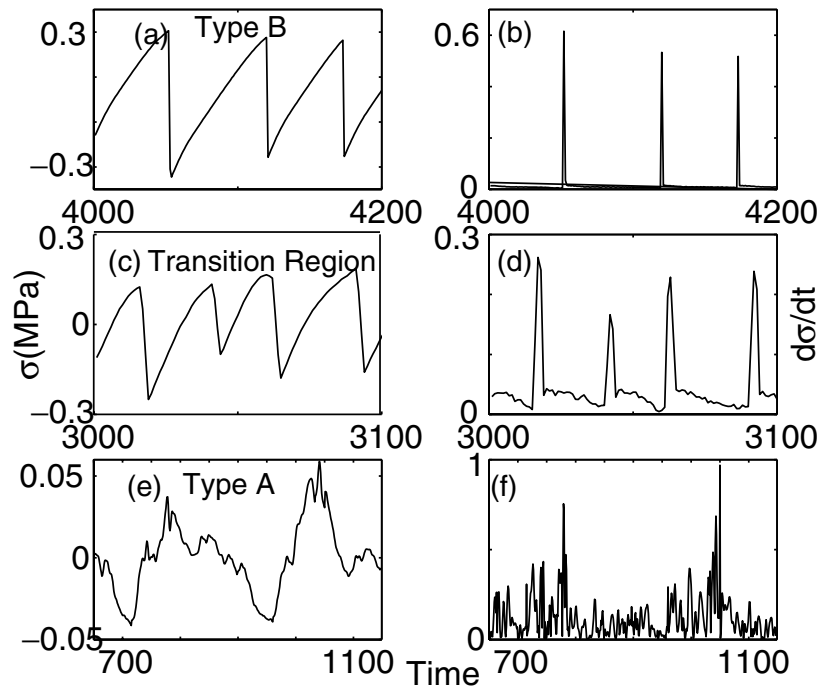

FIG. 1. Stress-time series at three strain rates: (a) $5.56 \times$ $10^{-6} \mathrm{~s}^{-1}, \quad$ (c) $2.8 \times 10^{-4} \mathrm{~s}^{-1}, \quad$ and (e) $5.56 \times 10^{-3} \mathrm{~s}^{-1}$. (b),(d), and (f) are the corresponding plots of $|d \sigma / d t|$. defined as the fraction of the pairs of vectors $\left(\vec{\xi}_{i}, \vec{\xi}_{j}\right)$ closer than a specified value $r$. The self-similar structure of the attractor, when it exists, is revealed by the scaling relation $C(r) \sim r^{\nu}$ in the limit of small $r$. As the embedding dimension $d$ is increased, the slope $\ln C(r) / \ln r$ tends to a constant value taken as the correlation dimension. The Lyapunov spectrum is computed using Eckmann's algorithm [15], suitably modified for short noisy time series [7]. In our algorithm, the number of neighbors of a vector $\vec{\xi}_{i}$ contained in a shell centered on $\vec{\xi}_{i}$ (whose size $\epsilon_{s}$ is measured as a percentage of the size of the attractor) is large enough to properly sample the statistics of uncorrelated noise corrupting the original signal. We consider the dynamics to be deterministic if a fair range of $\epsilon_{s}$ can be found such that stable values (i.e., constant in that range) emerge for a positive exponent and a zero exponent [7].

In the region of strain rates $5.6 \times 10^{-6} \leq \dot{\epsilon}_{a} \leq 1.4 \times$ $10^{-4} \mathrm{~s}^{-1}$, the data sets typically contain 10000 to 12000 points. The band patterns are of type $B$. We illustrate the results with the data file at $\dot{\epsilon}_{a}=5.6 \times 10^{-6} \mathrm{~s}^{-1}$. Figure 2 shows the $\log -\log$ plot of $C(r)$ for $d=15$ to 18 using $\tau=8$. The slopes are seen to converge to a value $\nu \sim 4.6$ for $d=17$ and 18 in the range $-4.9<\ln r<-3.2$.

The Lyapunov spectrum shown in Fig. 3 has been calculated using $d=5$. Stable positive and zero Lyapunov exponents are seen in the range $6 \%<\epsilon_{s}<12 \%$. The Lyapunov dimension $D_{\mathrm{KY}}$ obtained from the spectrum by using the Kaplan-Yorke conjecture turns out to be $D_{\mathrm{KY}} \approx$ $4.6=\nu$. Therefore, we conclude that the time series is of chaotic origin. Similar results were obtained with other samples in this region of $\dot{\epsilon}_{a}$, with values of $\nu$ and $D_{\mathrm{KY}}$ in the range 4.4 to 4.6 . In contrast, the correlation dimension did not converge and no positive Lyapunov exponent could be found for the data sets at higher $\dot{\boldsymbol{\epsilon}}_{a}$, implying that the dynamics is no more chaotic in that region of strain rate. In these cases, only the largest Lyapunov exponent could be calculated since the data contain only 3000 to 5000 points.

The quantity $|d \sigma / d t|$ clearly reflects the bursts of plastic activity (see Fig. 1). Therefore, we use its finite difference

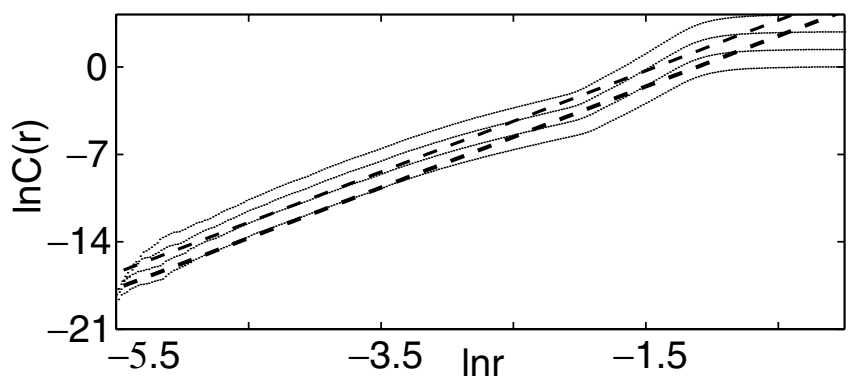

FIG. 2. Correlation integral $C(r)$ using $d=15$ to $18, \tau=8$ for $\dot{\epsilon}_{a}=5.56 \times 10^{-6} \mathrm{~s}^{-1}$. The curves corresponding to $d=$ 15 to 17 have been displaced with respect to $d=18$ by a constant amount. Dashed lines are guides to the eye. 


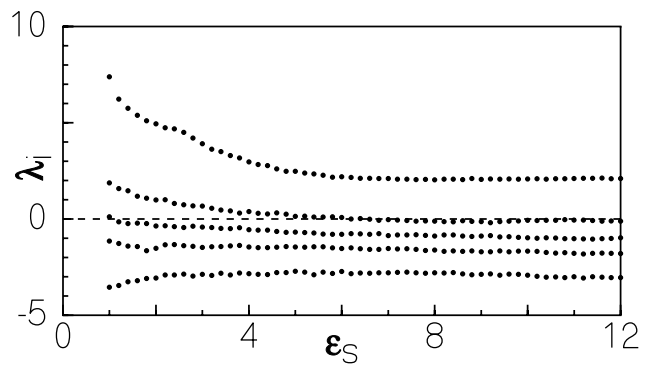

FIG. 3. Lyapunov exponents vs shell size $\epsilon_{s}$ for applied strain rate $\dot{\epsilon}_{a}=5.56 \times 10^{-6} \mathrm{~s}^{-1}$; embedding dimension $d=5$.

approximant $\psi_{i}\left(t_{i}\right)$ for statistical and multifractal analysis. Let $\Delta \psi$ denote the amplitude of the bursts and $\Delta t$ their durations. We investigate the distributions $D(\Delta \psi)$ of $\Delta \psi$ and $D(\Delta t)$ of $\Delta t$. Plots of $D(\Delta \psi)$ are shown in Fig. 4 for appropriately chosen values of $\dot{\epsilon}_{a}$. Peaked distributions are seen in the chaotic regime $\left(5.6 \times 10^{-6} \mathrm{~s}^{-1}\right.$ to $1.4 \times 10^{-4} \mathrm{~s}^{-1}$ ) indicating the existence of characteristic values. Now we turn to higher strain rates. On the basis of single crystal results, we expect to find SOC state. In SOC type dynamics, power law statistics arises when spatially extended driven systems naturally evolve to a critical state. On increasing $\dot{\epsilon}_{a}$, the distributions become broader and asymmetrical in the midregion of $\Delta \psi$, eventually leading to power law distributions (Fig. 4c).

For the data set at $\dot{\epsilon}_{a}=5.6 \times 10^{-3} \mathrm{~s}^{-1}$, the distribution has the form $D(\Delta \psi) \sim \Delta \psi^{-a}$ over 1 order of magnitude in $\Delta \psi$ with $a \sim 1.5$. Similarly, we find a power law distribution $D(\Delta t) \sim \Delta t^{-b}$ for the duration of the bursts with $b \sim 3.2$ and for the conditional average $\langle\Delta \psi\rangle_{c} \sim$ $\Delta t^{x}$ with $x \sim 4.2$. Thus, the scaling relation $x(a-1)+$ $1=b$ that characterizes SOC dynamics is well satisfied. Similar results are also found at the highest strain rate $\dot{\epsilon}_{a}=1.4 \times 10^{-2} \mathrm{~s}^{-1}$. Thus, the region of SOC-type dynamics extends from $\dot{\epsilon}_{a}=5.6 \times 10^{-3} \mathrm{~s}^{-1}$ onward, coinciding with the region of type $A$ bands.

Recall that a multifractal analysis was used to quantify the broad distribution of length scales occurring in the transition region between localized and delocalized states in the Anderson transition [13]. In our case also, there is a broad distribution of time scales in the region separating chaos and SOC (Fig. 4b). Thus, we anticipate that multifractal analysis will quantify this heterogeneity [16]. Let $N=N(\delta t)$ be the number of time intervals $\delta t$
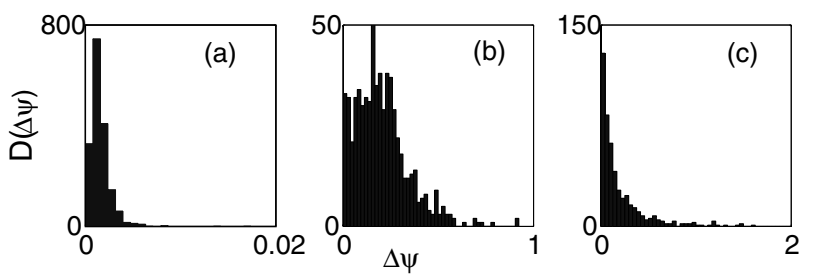

FIG. 4. Distributions $|D(\Delta \psi)|$ for the data sets at (a) $\dot{\epsilon}_{a}=$ $5.56 \times 10^{-6} \mathrm{~s}^{-1}$, (b) $1.4 \times 10^{-3} \mathrm{~s}^{-1}$, and (c) $5.56 \times 10^{-3} \mathrm{~s}^{-1}$. with $m$ points, required to cover $[0, K]$. Then, the normalized amplitude of the bursts in the $i$ th interval $\delta t$ is $p_{i}(\delta t)=\sum_{k=1}^{m} \psi_{i m+k} / \sum_{j=1}^{K} \psi_{j}$, usually called the probability measure. A conventional fractal is adequately described by a single scaling exponent, $D_{f}$, its fractal dimension, through $N(\delta t) \sim \delta t^{-D_{f}}$. However, heterogeneous sets require a continuum of scaling indices introduced via the probability $p_{i}(\delta t) \sim \delta t^{\alpha}$, where $\alpha$ is the strength of the local singularity. Then, $N(\delta t)$ generalizes to $N_{\alpha}(\delta t) \sim \delta t^{-f(\alpha)}$, where $f(\alpha)$ is the fractal dimension of the subset of intervals characterized by the exponent $\alpha$ [16]. The nonuniformity of the measure is captured by the range of multifractality $\theta=\alpha_{\max }-\alpha_{\min }$, where $\alpha_{\min }$ and $\alpha_{\max }$ are the extreme values of $\alpha$. Using another measure $\mu_{i}(\delta t, q)=p_{i}^{q} / \sum_{j} p_{j}^{q}$, where $q$ is a real number, $\alpha$ and $f(\alpha)$ can be directly calculated through

$$
\alpha=\lim _{\delta t \rightarrow 0} \frac{\sum_{i} \mu_{i}(\delta t, q) \ln p_{i}(\delta t)}{\ln \delta t}
$$

and

$$
f(\alpha)=\lim _{\delta t \rightarrow 0} \frac{\sum_{i} \mu_{i}(\delta t, q) \ln \mu_{i}(\delta t, q)}{\ln \delta t} .
$$

This canonical method was shown to be suitable for the analysis of short experimental data sets [17]. The spectrum $(\alpha, f(\alpha))$ is shown in Fig. 5 for $\dot{\epsilon}_{a}=1.4 \times 10^{-3} \mathrm{~s}^{-1}$.

The dependence of the multifractal range $\theta$ on $\dot{\epsilon}_{a}$ is shown in Fig. 6. Also displayed are the different dynamical regimes together with the band types. $\theta$ is seen to have relatively low values at both low and high strain rates. The small value of $\theta$ in the chaotic regime is due to the sharp peak in $D(\Delta \psi)$ (Fig. 4a), while that in the SOC region is due to the scaling nature of the distribution (Fig. 4c) [16]. In contrast, a sharp peak observed at intermediate $\dot{\epsilon}_{a}$ clearly signals a transition in the nature of dynamics from chaotic $B$-type to SOC $A$-type bands.

Distinct dynamical regimes and spatiotemporal patterns are displayed in the above analysis. Chaos is associated with type $B$ hopping bands at low applied strain rates, and SOC dynamics is present at high $\dot{\epsilon}_{a}$ in the domain of type $A$ propagating bands. The crossover from chaotic to SOC dynamics is clearly signaled by a burst in multifractality. Such a diversity in dynamics poses a challenge

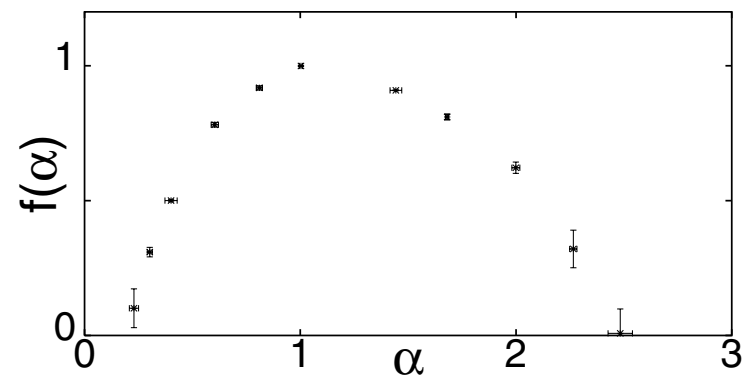

FIG. 5. Multifractal spectrum $(\alpha, f(\alpha))$ for the applied strain rate $\dot{\epsilon}_{a}=1.4 \times 10^{-3} \mathrm{~s}^{-1}, q \in[-5,+5]$. 


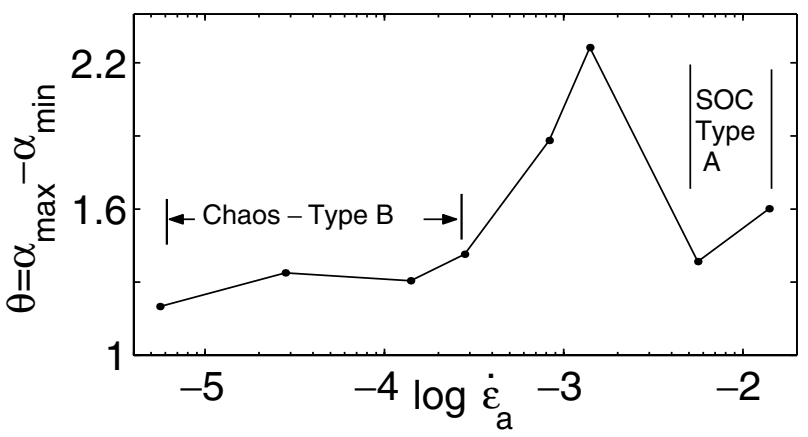

FIG. 6. The multifractal range $\theta$ vs applied strain rate $\dot{\epsilon}_{a}$. Regions of chaotic type $B$ and SOC type $A$ bands are marked.

for modeling the PLC effect. What needs to be understood is why the spatial correlations between bands and the complexity of the dynamics increase with increasing $\dot{\epsilon}_{a}$. The following arguments are quite general and encompass various mechanisms that have been proposed to explain the spatial coupling between bands (see [4,8]). A band of localized deformation induces long range stresses that favor strain uniformization in its neighborhood. However, during jerky flow (i.e., even in the presence of solute atoms) relaxation of these internal stresses is observed [18]. It occurs by thermally activated rearrangements of dislocation microstructure. Thus, both the magnitude and spatial extent of internal stresses are expected to decrease with time once a band is formed. Let $t_{r}$ denote this characteristic relaxation time.

At very low strain rates, the reloading time between two successive drops, $t_{l}$, is very large and $t_{l} \gg t_{r}$. The internal stresses are fully relaxed and no spatial correlation between bands is observed. This corresponds to the domain of type $C$ bands, which are not studied here. The absence of spatial correlation should make it easier to build a model based on the temporal mechanism of the instability, namely the repeated pinning and unpinning of dislocations in the field of solute atoms.

At larger strain rates, the reloading time $t_{l}$ decreases and becomes commensurate with the relaxation time $t_{r}$. Internal stresses are not totally relaxed and favor the formation of new bands nearby the previous ones, hence the hopping character of the associated type $B$ bands. At high $\dot{\epsilon}_{a}$, $t_{l} \ll t_{r}$, very little plastic relaxation occurs between the stress drops. Further, the reloading rates become increasingly a significant fraction of the unloading rates. Thus, the stresses felt by dislocations always remain close to the threshold for unpinning from the solute atoms. New bands can be formed in the field of unrelaxed internal stresses and recurrent partial events can overlap resulting in a hierarchy of length scales. This leads to both SOC dynamics and type $A$ propagating bands.

Models incorporating the basic instability mechanism and a spatial coupling $[4,8]$ seem to reproduce the various types of bands and the corresponding stress drop distributions. This suggests that they have the right basis for reproducing the corresponding dynamical regimes, chaos, and SOC, as well as the crossover between them.

In summary, a connection is made between the spatial patterns and the dynamical regimes associated with different strain rates in the jerky flow of polycrystals. A qualitative interpretation is proposed in terms of competing mechanisms that operate at different scales, local and global, and involve nonlocal effects. The crossover between the hopping bands associated with chaos and the propagating bands identified with the SOC state is well detected by a surge in the multifractal behavior of the distribution of plastic events. To the best of our knowledge, this is the first example where a crossover from localized to propagating states has been detected using a multifractal analysis based on purely experimental signals.

Support by CNRS and JNCASR under PICS No. 657 is gratefully acknowledged. G. A. and M. L. are grateful for the support of Metz University for their stays during 1998-2000 and G. A. for the partial support of Department of Science and Technology, Government of India.

*Corresponding author.

Email address: garani@mrc.iisc.ernet.in

[1] A. Portevin and F. Le Chatelier, C.R. Acad. Sci. Paris 176, 507 (1923).

[2] A. H. Cottrell, Dislocations and Plastic Flow in Crystals, (University Press, Oxford, 1953); J. Friedel, Dislocations (Pergamon Press, Oxford, 1964).

[3] A. van den Beukel, Phys. Status Solidi (a) 30, 197 (1975).

[4] M. Lebyodkin et al., Acta Mater. 48, 2529 (2000).

[5] G. Ananthakrishna et al., Scr. Metall. Mater. 32, 1731 (1995); S. Venkadesan, K. P. N. Murthy, S. Rajasekhar, and M. C. Valsakumar, Phys. Rev. E 56, 611 (1996).

[6] S. J. Noronha et al., Int. J. Bifurcation Chaos Appl. Sci. Eng. 7, 2577 (1997).

[7] G. Ananthakrishna, S. J. Noronha, C. Fressengeas, and L. P. Kubin, Phys. Rev. E 60, 5455 (1999).

[8] M. Lebyodkin, Y. Bréchet, Y. Estrin, and L. P. Kubin, Phys. Rev. Lett. 74, 4758 (1995).

[9] G. D’Anna and F. Nori, Phys. Rev. Lett. 85, 4096 (2000).

[10] G. Ananthakrishna and M. C. Valsakumar, J. Phys. D 15, L171 (1982).

[11] P. Bak, C. Tang, and K. Wiesenfeld, Phys. Rev. A 38, 364 (1988); Phys. Rev. Lett. 59381 (1987).

[12] P. W. Anderson, Phys. Rev. 109, 1492 (1958).

[13] M. Schreiber and H. Grussbach, Phys. Rev. Lett. 67, 607 (1991).

[14] P. Grassberger and I. Procaccia, Physica (Amsterdam) 9D, 189 (1983).

[15] J. P. Eckmann, S. O. Kamphorst, D. Ruelle, and S. Ciliberto, Phys. Rev. A 34, 4971 (1986).

[16] T. C. Halsey et al., Phys. Rev. A 33, 1141 (1986).

[17] A. B. Chhabra and R. V. Jensen, Phys. Rev. Lett. 62, 1327 (1989).

[18] K. Chihab, Y. Estrin, L. P. Kubin, and J. Vergnol, Scr. Metall. 21, 203 (1987). 